\section{Sleuthing for toxicity}

By Tracey Baas, Senior Editor

Immunocore Ltd., Adaptimmune Ltd. and University of Pennsylvania researchers have determined that off-target toxicity most likely caused the two fatalities in a cancer trial of affinity-enhanced $\mathrm{T}$ cell receptors. ${ }^{1}$ The results highlight the need in the cancer immunotherapy space for harnessing biologically appropriate cellular testing and deep molecular analysis approaches to minimize cross-reactivity-related toxicity in future adoptive immunotherapy trials.

A primary challenge in engineering $\mathrm{T}$ cells for adoptive immunotherapy is selecting a cancer-associated antigen that is absent on healthy tissues and lacks homology to other self-proteins.

Over the last few years, melanoma-associated antigen A3 (MAGEA3) emerged as one of the most promising targets for adoptive immunotherapy because it is highly expressed in a number of tumors and its expression in healthy individuals is limited to the testes. ${ }^{2,3}$

Because the human immune system has evolved to avoid the generation of high-affinity $\mathrm{T}$ cell receptors (TCRs) against selfproteins to preclude autoimmune reactions, endogenous TCRs must be optimized for cancer antigen affinity. On the other hand, engineering TCRs with enhanced affinity for specific tumor antigens presents the challenge of minimizing affinity for other homologous self-proteins to prevent nonspecific binding of the TCRs.

Between 2008 and 2010, an Immunocore, Adaptimmune and UPenn team led by Carl June designed affinity-enhanced TCRs that target a nine-amino-acid MAGEA3 peptide. In functional analyses, a TCR called a3a was identified as the most potent MAGEA3-specific TCR, and extensive preclinical studies with a3a-transduced T cells showed no signs of toxicity.

June is a professor in the Department of Pathology and Laboratory Medicine at the Perelman School of Medicine at the University of Pennsylvania and director of the translational research program at the Abramson Family Cancer Research Institute at the University of Pennsylvania.

However, when the a3a-engineered $\mathrm{T}$ cells were used in two patients with melanoma or myeloma, both died of acute cardiac failure within five days of receiving the therapy. ${ }^{4}$ Analysis of autopsy samples showed $\mathrm{T}$ cell infiltration in the hearts of both patients but no MAGEA3 expression, suggesting off-target toxicity.

Earlier this year, a National Cancer Institute (NCI)-led Phase I/II trial in patients with metastatic cancer given an affinity-enhanced TCR targeting three MAGEAs-MAGEA3, MAGEA9 and MAGEA12resulted in fatal neurotoxicity in two of nine patients. ${ }^{5}$ In that instance, analysis of autopsy brain samples using real-time quantitative PCR, nanostring quantitation and deep sequencing showed that one of the
antigens-MAGEA12-was expressed in the brain, indicating that toxicity may have stemmed from on-target effects in nontumor tissue.

This trial was led by Steven Rosenberg, chief of the surgery branch and head of the tumor immunology section at the NCI.

Although the NCI study showed that affinity-enhanced T cells could hit a target expressed in nontumor tissue at such low levels that they would be missed in standard preclinical studies, this did not solve the mystery of the UPenn study-quantitative RT-PCR confirmed the absence of MAGEA3 in both patients' hearts.

Now, Immunocore, Adaptimmune and UPenn researchers, led by Bent Jakobsen, CSO of Immunocore, have uncovered the cause of infiltration by the a3a-engineered $\mathrm{T}$ cells and ensuing cardiac toxicity using functional analysis and amino acid scanning.

In 38 normal, cardiac-derived primary cells, the a3a-engineered $\mathrm{T}$ cells caused no $\mathrm{T}$ cell activation.

Next, the group explored a more biologically relevant cardiac cell culture-iCell cardiomyocytes from Cellular Dynamics International Inc. Unlike primary cells plated in a dish, iCell cardiomyocytes show biochemical, electrophysiological and mechanical characteristics of heart tissue, including spontaneous beating.

The cells did not show MAGEA3 expression. Nevertheless, the a3aengineered $\mathrm{T}$ cells killed the iCell cardiomyocytes within 24 hours, suggesting something other than MAGEA3 was activating the T cells.

To identify what the $\mathrm{T}$ cells were targeting, each amino acid in the nine-amino-acid MAGEA3 peptide was replaced in turn with either alanine or glycine residues. The process allowed the researchers to discover a motif essential for a 3 a TCR binding within that nineamino-acid peptide. A search of genome databases found a trio of proteins that harbor that motif, and the group performed multiple cell line studies to narrow the culprit to the muscle protein titin (TTN).

Notably, titin activated MAGEA3-targeting T cells in human cell lines but not in mouse cell lines, suggesting preclinical toxicity testing would not have revealed the off-target effects.

Expression of titin was confirmed in 3D beating cultures of iCell cardiomyocytes and in five independent human cardiac tissue samples. The results suggest a3a-engineered $\mathrm{T}$ cells recognize a sequence motif within titin, resulting in off-target toxicity.

Results were published in Science Translational Medicine.

"The iPS [induced pluripotent stem] cell system that the authors have used is a beautiful way to demonstrate the reactivity of the TCRmodified cells against titin-expressing cells. In combination with the amino acid scanning approach, this appears a viable strategy to identify possible cases of off-target reactivity before they appear in the clinic," said Ton Schumacher, a professor and group leader in immunology at The Netherlands Cancer Institute.

The findings should extend beyond titin and could be used to filter out engineered TCRs that might interact with proteins they were not designed to target.

\section{Rooting out safety issues}

Schumacher cautioned that because the procedure relies on primary sequence homology between the target epitope of interest and the 
cross-reactive epitope, "a fraction of potential off-targets may be missed" that are due to secondary structural homologies.

Researchers also thought that a deeper understanding of how the enhanced affinity of TCRs can lead to off-target toxicity was required.

"The observed off-target possibility was due to the in vitro affinity maturation, and the use of TCR identification systems that do not rely on in vitro affinity maturation would provide a nice way around the issue," noted Schumacher.

"Cameron and colleagues provide clear evidence that TCR degeneracy of antigen recognition represents a true risk for clinical TCR gene therapy. The chances that such degenerate recognition leads to autoimmunity, as in the case of the reported cardiotoxicity, significantly increases when TCRs have an enhanced affinity for their cognate antigen," said Reno Debets, an associate professor of experimental tumor immunology at Erasmus Medical Center. "Although I would not argue against the benefit of affinity enhancement of TCRs, I would advocate for studies to define the potential rules to safely mutate complementarity-determining regions within the TCRs, and investigating means to improve this gene therapy without enhancing the affinity of TCR, such as enhancement of $\mathrm{T}$ cell fitness and introduction of strategies to counteract the immune-suppressive micromilieu of the tumor."

Debets also said that it should become standard practice to test TCR-transduced T cells against 3D cultures of differentiated cells.

"Clearly these cultures, and not standard cultures of primary cells, better represent human organs with respect to their immunogenicity," he said.

"Inclusion of specialized cultures-representing, for instance, vital organs-as T cell targets further advances the safety tests of TCR gene therapy irrespective of whether TCRs are affinity enhanced or not."

Going forward, the Immunocore, Adaptimmune and UPenn team will use a cellular screening panel that will include primary cells, cells grown in $3 \mathrm{D}$ cultures and iCells that represent organs most likely to be affected.

"The tools we used to identify titin continue to be developed and are now being used to safety test our new TCRs," Nick Pumphrey, head of pipeline research at Adaptimmune, told SciBX. "To complement our cell testing, we also use RT-PCR data for expression analysis of potential targets and possible off-target peptides using different tissue samples, including brain sections. RT-PCR data can only tell you if the protein you are probing is present, whereas cell testing highlights on- or off-target toxicity regardless of whether the specificity is known or not."

Pumphrey told SciBX that one limitation with iCell neuronal culture is that it is made up of inhibitory $\gamma$-aminobutyric acid (GABA)-ergic and glutamatergic neurons. "There is a whole array of other neuronal types in the brain too, "he said. "We cannot rely on any one cell line as being representative of a complex tissue, and [we] are working to make our cell testing panels as broad as possible and combining this approach with RT-PCR and peptide scanning information to select the best TCRs to the best targets."

Mark Dudley, a staff scientist and head of the cell production facility at the NCI, noted that "retrospectively identifying the mechanism of a known toxicity is much easier, and a very different problem, than prospectively determining whether a TCR will be safe for adoptive therapy. It is unlikely that any combination of preclinical tests will completely predict the behavior of novel $\mathrm{T}$ cell receptors and chimeric antigen receptors introduced into T cells."

The upshot, he said, is that "engineered T cells are potent clinical agents, and some can only be fully evaluated by testing their antitumor effects in patients."

Chiara Bonini, head of the Experimental Hematology Unit and a member of the cancer immunotherapy and gene therapy program and the bone marrow transplantation unit of the San Raffaele Scientific Institute, said measures to minimize toxicity could include "dose escalation of infused $\mathrm{T}$ cells, which could allow detection of toxicity of a milder and more controllable grade before increasing the $\mathrm{T}$ cell dose; nuclease-mediated knockdown of the endogenous TCR to avoid TCR mispairing and alloreactivity; and the incorporation of suicide genes, which might allow the elimination of activated $\mathrm{T}$ cells as soon as toxicity is observed."

Debets was unsure about the suicide gene approach. "Although the suicide gene could provide the option to delete TCR-transduced T cells from patients once there are signs of toxicity, it is questionable whether such a switch could counteract the fast kinetics of toxicity reported in this study - four to five days following T cell infusion," he said.

Michel Sadelain, director of Memorial Sloan-Kettering Cancer Center's Center for Cell Engineering, also was unsure about the suicide gene in such a rapidly fatal context. He thinks that an alternative safety feature would be to use $\mathrm{T}$ cells that transiently express the affinityenhanced TCRs.

"The T cells can be transfected with mRNA that codes for the TCR, thereby generating T cells that have a limited lifetime within the recipient's tissues. Such T cells, on the other hand, would have a reduced therapeutic impact if adminstered only once," he said.

"The new study shows that strategies needed to evaluate TCRinduced toxicities will need to be broadened and more labor intensive," added Sadelain. "And in the end, these strategies may still not be enough to identify all potential risks. Fast-acting suicide genes should prove to be useful. Using $\mathrm{T}$ cells that are self-limiting and truly selective is the only way to truly guard patients from off-target or on-target off-tissue toxicities when moving TCRs into first-in-man trials."

Adaptimmune is recruiting patients with multiple myeloma and synovial sarcoma for trials using affinity-enhanced TCR-expressing T cells that target cancer/testis antigen 1B (CTAG1B; NY-ESO-1). The company said its products have shown no off-target toxicity and encouraging early data.

Immunocore is focused on the discovery and development of TCRbased therapies to treat cancer and viral diseases using their immunemobilizing, monoclonal TCRs against cancer (ImmTACs). ImmTACs consist of a very high-affinity TCR linked to a CD3 receptor, a strong $\mathrm{T}$ cell activator. 


\section{ANALYSIS}

Currently, UPenn is concentrating its efforts on clinical trials with chimeric antigen receptor-expressing T cells with Novartis AG, and Adaptimmune has entered into a cell therapy manufacturing services agreement with NeoStem Inc.'s Progenitor Cell Therapy subsidiary to enable their new clinical trials with affinity-enhanced TCR-expressing $\mathrm{T}$ cells to be run independently of UPenn.

Baas, T. SciBX 6(33); doi:10.1038/scibx.2013.879

Published online Aug. 29, 2013

\section{REFERENCES}

1. Cameron, B.J. et al. Sci. Transl. Med.; published online Aug. 7, 2013; doi:10.1126/scitranslmed.3006034

Contact: Bent K. Jakobsen, Immunocore Ltd., Abingdon, U.K. e-mail: bent.jakobsen@immunocore.com

2. Caballero, O.L. \& Chen, Y.-T. et al. Cancer Sci. 100, 2014-2021 (2009)

3. Gaugler, B. et al. J. Exp. Med. 179, 921-930 (1994)

4. Linette, G.P. et al. Blood 122, 863-871 (2013)
5. Morgan, R.A. et al. J. Immunother. 36, 133-151 (2013)

\section{COMPANIES AND INSTITUTIONS MENTIONED}

Abramson Family Cancer Research Institute at the University of Pennsylvania, Philadelphia, Pa.

Adaptimmune Ltd., Abingdon, U.K.

Cellular Dynamics International Inc. (NASDAQ:ICEL), Madison, Wis. Erasmus Medical Center, Rotterdam, the Netherlands Immunocore Ltd., Abingdon, U.K.

Memorial Sloan-Kettering Cancer Center, New York, N.Y.

National Cancer Institute, Bethesda, Md.

NeoStem Inc. (NASDAQ:NBS), New York, N.Y.

Novartis AG (NYSE:NVS; SIX:NOVN), Basel, Switzerland

The Netherlands Cancer Institute, Amsterdam,

the Netherlands

Perelman School of Medicine at the University of Pennsylvania,

Philadelphia, Pa.

San Raffaele Scientific Institute, Milan, Italy

University of Pennsylvania, Philadelphia, Pa. 